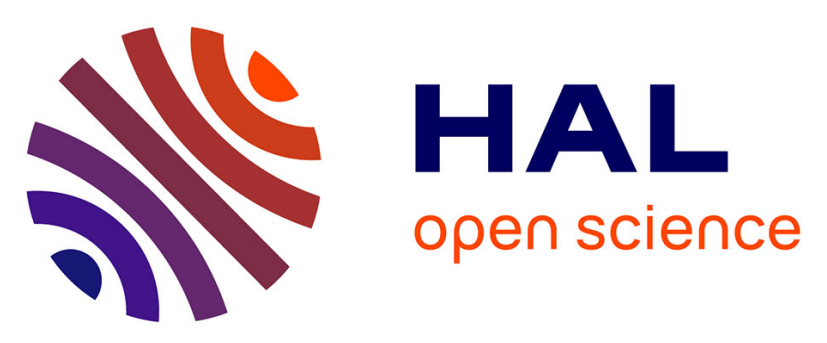

\title{
Effect of viscoelasticity on quality factor of microcantilever chemical sensors: optimal coating thickness for minimum limit of detection
}

Frédéric Lochon, Isabelle Dufour, Dominique Rebiere, Uttara Sampath, Stephen Heinrich, Fabien Josse

\section{To cite this version:}

Frédéric Lochon, Isabelle Dufour, Dominique Rebiere, Uttara Sampath, Stephen Heinrich, et al.. Effect of viscoelasticity on quality factor of microcantilever chemical sensors: optimal coating thickness for minimum limit of detection. IEEE Sensors 2005, 2005, Irvine, United States. pp.265-268. hal00182217

\section{HAL Id: hal-00182217 https://hal.science/hal-00182217}

Submitted on 15 Jan 2008

HAL is a multi-disciplinary open access archive for the deposit and dissemination of scientific research documents, whether they are published or not. The documents may come from teaching and research institutions in France or abroad, or from public or private research centers.
L'archive ouverte pluridisciplinaire HAL, est destinée au dépôt et à la diffusion de documents scientifiques de niveau recherche, publiés ou non, émanant des établissements d'enseignement et de recherche français ou étrangers, des laboratoires publics ou privés. 


\section{Effect of Viscoelasticity on Quality Factor of Microcantilever Chemical Sensors: Optimal Coating Thickness for Minimum Limit of Detection}

\author{
Lochon F., Dufour I., Rebière D. \\ IXL Laboratory, CNRS UMR5818, \\ ENSEIRB/Université Bordeaux 1, 351 cours de la Libération, \\ 33405 Talence Cedex, France, \\ dufour@ixl.fr
}

\author{
Sampath U., Heinrich S.M., Josse F. \\ Marquette University, \\ P.O.Box 1881, Milwaukee, \\ WI 53201-1881, USA, \\ stephen.heinrich@marquette.edu \\ fabien.josse@marquette.edu
}

\begin{abstract}
Microcantilevers with polymer coatings hold great promise as resonant chemical sensors. It is known that the coated cantilever sensitivity increases with coating thickness; however, the drawback of increasing the coating thickness is the increase of the frequency noise and thus the deterioration of the sensor's limit of detection. In this paper, an analytical expression for the viscoelastic losses in the coating, hence the quality factor, is established and is used to explain the observed increase of the frequency noise with the polymer thickness. This result is then used to demonstrate that an optimum coating thickness exists that will minimise the limit of detection.
\end{abstract}

\section{INTRODUCTION}

Resonant microcantilever-based sensors have emerged as a new sensitive detection technique. In chemical sensing applications, the device consists of a microcantilever and a chemically-sensitive coating, which absorbs the molecule of interest (Fig. 1). The absorbed molecules can then be detected by monitoring the mechanical resonant frequency.

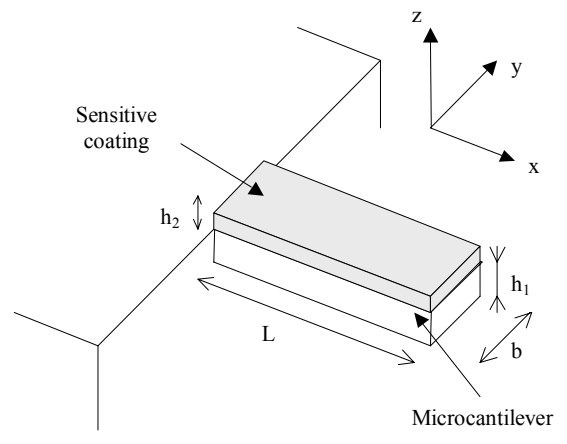

Figure 1. Geometry of the cantilever and its sensitive coating

The choice of the coating thickness for minimum limit of detection (LOD) is known to be a difficult task because of its different effect on sensor characteristics. In fact, the sensor sensitivity increases with coating thickness whereas the frequency noise also increases. The aim of this paper is to demonstrate through rigorous analysis of a hybrid (elastic/viscoelastic) beam that there exists an optimal coating thickness leading to the minimal LOD. An analytical expression showing the effect of the viscoelasticity of the layer on the sensor quality factor, $Q$, is first established. Using this expression, the dependence of the LOD on the coating thickness is derived for a resonant frequency-based sensor for the cases of (1) intrinsic noise and (2) operation within an oscillator configuration. Theoretical results are then compared with preliminary measurements.

\section{EFFECT OF SENSITIVE LAYER VISCOELASTICITY ON SENSOR QUALITY FACTOR}

An expression for the sensitivity $S$ of microcantileverbased chemical sensors [1], is given by (1) and is plotted in Fig. 2:

$$
S=\frac{\Delta f}{C_{g}}=\frac{K h_{2} f_{r e s}}{2\left(\rho_{1} h_{1}+\rho_{2} h_{2}\right)}
$$

where $f_{\text {res }}$ is the resonant frequency, $\Delta f$ is the frequency shift due to analyte sorption, $K$ is the partition coefficient of the coating/analyte pair in the fluid environment, $h_{l}$ and $h_{2}$ are the microcantilever and sensitive coating thicknesses, and $\rho_{1}$ and $\rho_{2}$ are the respective mass densities. According to (1), the sensitivity may be improved by increasing the thickness, $h_{2}$, of the polymeric sensitive coating, provided that $\rho_{2} h_{2}<2 \rho_{1} h_{1}[1]$.

Although measurements (see section IV and [2]) have shown that increasing the sensitive coating thickness improves sensitivity, a corresponding decrease in Q is also observed. These effects adversely influences the sensor's frequency noise and, thus, its LOD. 
If only viscous losses [3] due to damping in the gas environment are considered, the quality factor increases with added mass. So, the observed decrease of the quality factor has to be explained by taking into account another loss phenomenon. It is hypothesized that this decrease is primarily due to internal losses in the viscoelastic sensitive coating.

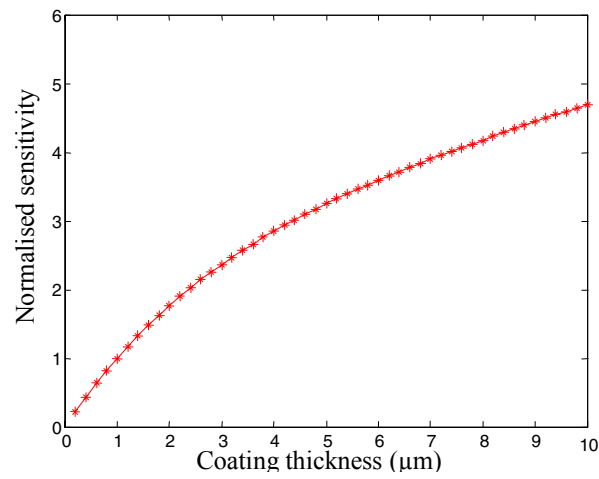

Figure 2. Normalized sensitivity versus coating thickness (PIB coating, silicon cantilever $4 \mu \mathrm{m} \times 50 \mu \mathrm{m} \times 300 \mu \mathrm{m})$. Normalization: $S=1$ for $h_{2}=1 \mu \mathrm{m}$.

\section{A. Viscoelastic Losses}

The hybrid (elastic/viscoelastic) beam can be assumed to be replaced by an equivalent homogeneous viscoelastic beam [4] whose complex flexural rigidity, $(E I)^{*}$, is given as:

$$
(E I)^{*}=E_{1} I_{1}+E_{2}^{\prime}(\omega) I_{2}+j E_{2}^{\prime \prime}(\omega) I_{2}
$$

where $E_{1}$ is the Young's modulus of the elastic material and $E^{\prime}{ }_{2}+j E^{\prime \prime}{ }_{2}$ the complex Young's modulus of the viscoelastic sensitive layer. $I_{1}$ and $I_{2}$ are the moments of inertia of the elastic and viscoelastic beam layers given respectively by

$$
\begin{gathered}
I_{1}=\frac{b h_{1}^{3}}{12}+b h_{1}\left(h_{N}-h_{2}-\frac{h_{1}}{2}\right)^{2} \\
I_{2}=\frac{b h_{2}^{3}}{12}+b h_{2}\left(h_{N}-\frac{h_{2}}{2}\right)^{2}
\end{gathered}
$$

where $b$ is the beam's width. The geometric properties (3) and (4) are with respect to the average position of the timevarying neutral axis of the hybrid beam, which is given by

$$
h_{N}=\frac{h_{2}}{2}+\frac{h_{1} E_{1}}{2} \frac{\left(h_{1}+h_{2}\right)\left(h_{1} E_{1}+h_{2} E_{2}^{\prime}\right)}{\left(h_{1} E_{1}+h_{2} E_{2}^{\prime}\right)^{2}+h_{2}^{2} E_{2}^{\prime \prime 2}}
$$

The equation of motion of an harmonically excited hybrid beam considering the only loss mechanism to be associated with the loss modulus of the sensitive layer, is

$$
(E I)^{*} \frac{\partial^{4} w(x, t)}{\partial x^{4}}+m_{L} \frac{\partial^{2} w(x, t)}{\partial t^{2}}=F(x) e^{j \omega t}
$$

where $w(x, t)$ is the (complex and harmonically varying) transverse displacement, $F(x)$ is the arbitrary distribution of the force amplitude, $m_{L}$ is the mass per unit length of the beam and $\varpi$ is the angular forcing frequency. An analysis of the solution of (6) leads to an expression for the resonant frequency, $f_{\text {res }}$, and quality factor associated with the viscoelastic losses in the sensitive layer, [4]:

$$
\begin{aligned}
f_{\text {res }} & =\frac{1.875^{2}}{2 \pi L^{2}} \sqrt{\frac{E_{1} I_{1}+E_{2}^{\prime} I_{2}}{m_{L}}} \\
Q_{\text {viscoel }} & =\frac{1}{2\left(1-\sqrt{1-\frac{E_{2}^{\prime} I_{2}}{E_{1} I_{1}+E_{2}^{\prime} I_{2}}}\right)}
\end{aligned}
$$

For a typical silicon cantilever and a polymeric sensitive coating, $E_{2}^{\prime} \ll E_{1}$ and $E^{\prime \prime}{ }_{2} \ll E_{1}$. A first-order approximation to (8) yields

$$
Q_{\text {viscoel }} \approx \frac{h_{1}^{3}}{h_{2}\left(h_{2}^{2}+3\left(h_{1}+h_{2}\right)^{2}\right)} \frac{E_{1}}{E^{\prime \prime}}
$$

This expression clearly shows that the quality factor due to coating losses decreases with increasing coating thickness. In the case of a small coating thickness, $h_{2}<<h_{1}$, the same expression as that of surface losses developed in [5] and [6] is found. An advantage of (8) is that it is valid for arbitrary values of thicknesses and moduli for the hybrid beam.

\section{B. Total Sensor Quality Factor}

When a microcantilever resonates in a gas or liquid medium, there are different mechanical loss mechanisms: viscoelastic and thermoelastic losses in the microcantilever, viscous and acoustic losses in the surrounding medium, losses due to radiation of elastic waves at the support, etc. Each of these loss mechanisms has an associated quality factor $Q_{i}$ and the overall quality factor $Q_{\text {total }}$ is obtained by

$$
\frac{1}{Q_{\text {total }}}=\sum_{i} \frac{1}{Q_{i}}
$$

In the case of chemical sensors, the surrounding medium is usually either a gas at atmospheric pressure or a liquid. Consequently, without the sensitive coating, the dominant losses are due to viscous damping, $Q_{\text {viscous }}$, and the results of Sader [3] are then applicable. With the sensitive coating, both quality factors, $Q_{\text {viscous }}$ and $Q_{\text {viscoel }}$, may need to be 
considered. In Fig. 3, the two quality factors are plotted as a function of the viscoelastic layer thickness. The total quality factor, $Q_{\text {total }}$, is also plotted. These plots and other simulations in this paper are based on a poly(isobutylene) (PIB) coating with storage and loss moduli of $E^{\prime}{ }_{2}=429 \mathrm{MPa}$ and $E^{\prime \prime}{ }_{2}=650 \mathrm{MPa}$ at the resonant frequency of $58 \mathrm{kHz}[7,8]$. If only viscous damping is considered, the quality factor increases with the added mass of the sensitive layer. The observed decrease of the total quality factor can be explained by the existence of viscoelastic losses in the sensitive coating.

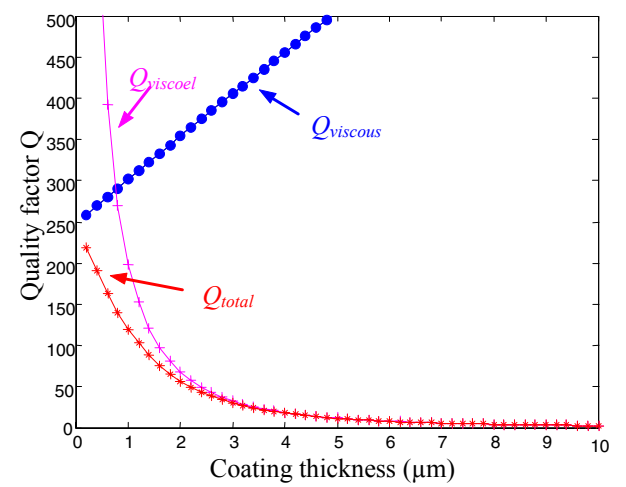

Figure 3. Quality factors versus coating thickness (PIB coating, silicon cantilever $4 \mu \mathrm{m} \times 50 \mu \mathrm{m} \times 300 \mu \mathrm{m})$.

\section{DEPENDENCE OF LIMIT OF DETECTION ON COATING THICKNESS}

The choice of a coating thickness that minimizes limit of detection (LOD) is known to be a difficult task because of the different effects the thickness has on sensor characteristics. In fact, as shown in section II, the sensor sensitivity increases with coating thickness whereas the total quality factor decreases; thus, the frequency noise increases. The aim of this section is to demonstrate using (8) that there exists an optimal coating thickness that minimizes the LOD. Two cases are studied: (1) the case of intrinsic noise which is observed in direct spectrum analysis, and (2) the case of operation within an oscillator configuration.

\section{A. Intrinsic Noise}

Here the focus is on the intrinsic noise mechanisms since they determine the ultimate limits of the sensor's performance. When a microcantilever is in an ambient thermal environment, there is a continuous exchange of the mechanical energy accumulated in the microcantilever and the thermal energy of the environment. This exchange results in spontaneous microcantilever oscillation. Due to this energy exchange, the resonant frequency $f_{\text {res }}$ is subjected to frequency fluctuations $\Delta f_{\text {noise_intrins }}$ given by [9],

$$
\Delta f_{\text {noise_intrins }}=\frac{1}{z_{\max }} \sqrt{\frac{2 \pi f_{\text {res }} k_{B} T B}{k Q_{\text {total }}}}
$$

where $k_{B}=$ Boltzmann constant, $T=$ absolute temperature, $B=$ measurement bandwidth, $k=$ microcantilever stiffness, and $z_{\max }=$ microcantilever oscillation amplitude.

The limit of detection is usually defined as the analyte concentration corresponding to a frequency shift equal to three times the frequency noise of the system measurement. Thus, (11) and (1) may be combined to yield the sensor's LOD when intrinsic noise is considered:

$$
L O D_{\text {noise_intrins }}=\frac{3 \Delta f_{\text {noise_intrins }}}{S} \propto \frac{\rho_{1} h_{1}+\rho_{2} h_{2}}{h_{2} \sqrt{k f_{\text {res }} Q_{\text {total }}}}
$$

In (12) only the terms depending on the coating thickness are kept in order to study the LOD dependence on the coating thickness. This expression allows one to determine the optimum sensitive coating thickness for minimum LOD.

The case of a PIB coating on a silicon microcantilever $4 \mu \mathrm{m} \times 50 \mu \mathrm{m} \times 300 \mu \mathrm{m}$ is presented in Fig. 4. Due to the viscoelastic effect, an optimum coating thickness exists, but if the coating thickness exceeds the optimal value, the resulting increase in LOD is not very significant.

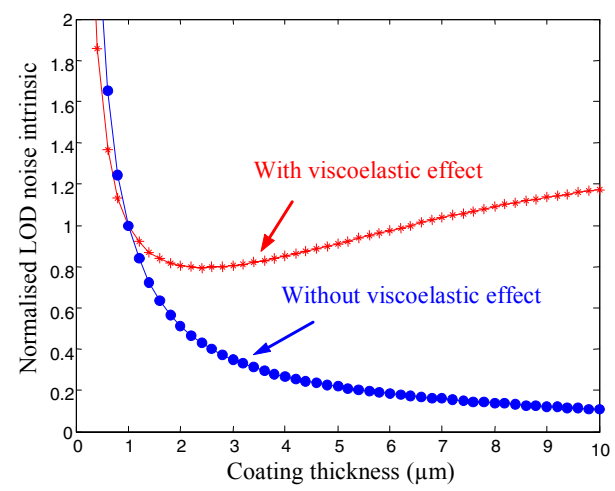

Figure 4. Normalised limit of detection versus coating thickness in the case of intrinsic noise (PIB coating, silicon cantilever $4 \mu \mathrm{m}$ x $50 \mu \mathrm{m} \times$ $300 \mu \mathrm{m})$. Normalization: $L O D=1$ for $h_{2}=1 \mu \mathrm{m}$.

\section{B. Oscillator Configuration}

Usually, in order to accurately measure the resonant frequency change, the microcantilever is inserted in the feedback loop of oscillator. The output signal is then the oscillaton frequency that can be measured with a counter. For a high degree of accuracy, the oscillator must be as stable as possible. The oscillator stability may be characterized by the frequency noise, $\Delta f_{\text {noise oscil }}$, which depends on the resonant frequency and the total quality factor as follows [10]:

$$
\Delta f_{\text {noise_oscil }} \propto \frac{f_{\text {res }}}{Q_{\text {total }}}
$$


As in the case of intrinsic noise, the frequency fluctuation $\Delta f_{\text {noise_oscil }}$ can be used to relate the LOD to $h_{2}$ :

$$
L O D_{\text {noise_oscil }}=\frac{3 \Delta f_{\text {noise_oscil }}}{S} \propto \frac{\rho_{1} h_{1}+\rho_{2} h_{2}}{h_{2} Q_{\text {total }}}
$$

Expression (14) may be used to determine the optimum sensitive coating thickness for minimum LOD. The case of a PIB coating on a silicon microcantilever $(4 \mu \mathrm{m}$ x $50 \mu \mathrm{m} \times$ $300 \mu \mathrm{m}$ ) is presented in Fig. 5. Clearly, when the viscoelastic losses are included, an optimum coating thickness exists. However, the oscillator configuration case differs significantly from the intrinsic noise case, in that an increase in coating thickness beyond the optimal value may seriously compromise the LOD. This phenomenon has been observed experimentally by Lange et al [2].

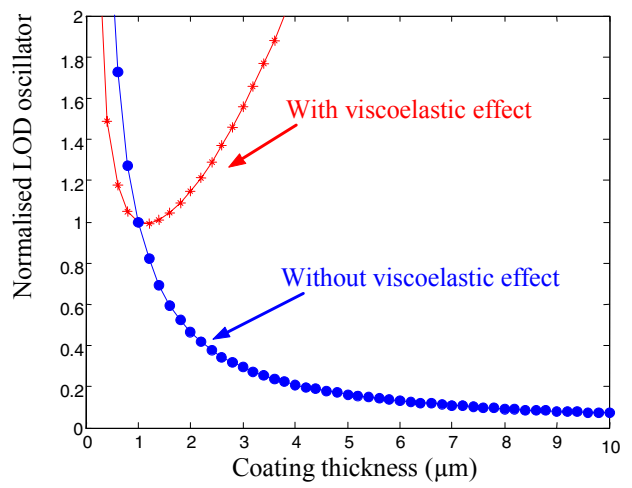

Figure 5. Normalised limit of detection versus coating thickness in the case of oscillator configuration (PIB coating, silicon cantilever $4 \mu \mathrm{m} \mathrm{x}$ $50 \mu \mathrm{m} \times 300 \mu \mathrm{m})$. Normalization: $L O D=1$ for $h_{2}=1 \mu \mathrm{m}$.

\section{EXPERIMENTAL RESULTS AND DISCUSSION}

In order to validate expression (8) for the quality factor associated with viscoelastic coating losses in the layer (which is the basis for determining the optimal coating thickness), some preliminary measurements have been made on a silicon microcantilever ( $L=6000 \mu \mathrm{m}, b=200 \mu \mathrm{m}, h_{1}=$ $221 \mu \mathrm{m})$ for which $f_{\text {res }}=7.3 \mathrm{kHz}$ and $Q_{\text {total }}=1686$. A PIB coating was sprayed onto the microcantilever and the quality factor was measured with a gain/phase analyzer (HP 4194A) for different coating thicknesses. The measurements are presented in Fig. 6. The modeling results with and without the viscoelastic losses (at $7.3 \mathrm{kHz}, E_{2}^{\prime}=11.4 \mathrm{MPa}$ and $\left.E "_{2}=19.6 \mathrm{MPa}[7,8]\right)$ are also shown.

Regarding Fig. 6, it clearly appears that, even if the viscoelastic quality factor (8) is very large (more than 27,500 here) compared to the total quality factor (less than 1686), the viscoelastic loss mechanism is the primary factor responsible for the observed decrease in the (total) quality factor. This being the case, one may use (12) or (14) and the relationship between quality factor and coating thickness (using (8) or measurements) to estimate the optimal coating thickness in order to achieve the minimum limit of detection.

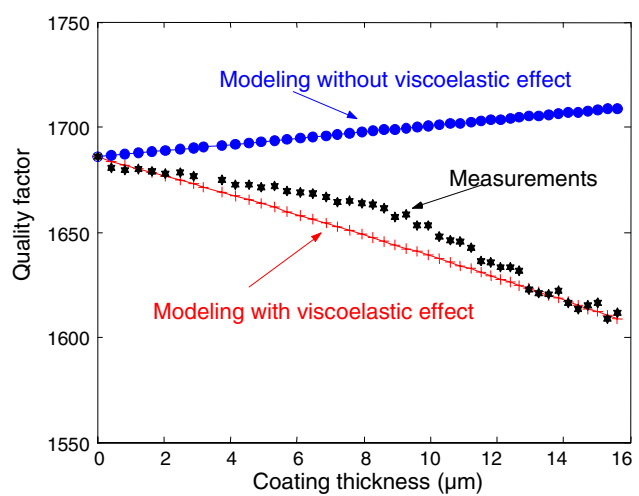

Figure 6. Total quality factor versus coating thickness (PIB coating, silicon cantilever $221 \mu \mathrm{m}$ x $200 \mu \mathrm{m}$ x $6 \mathrm{~mm}$ ). Measurements and modeling (with and without viscoelastic losses)

\section{ACKNOWLEDGMENT}

This work was sponsored in part by the French Agency for Environment and Energy Management (ADEME) and the 'Région Aquitaine'.

\section{REFERENCES}

[1] F. Lochon, I. Dufour, D. Rebière, "An alternative solution to improve sensitivity of resonant microcantilever chemical sensors: comparison between using high-order modes and reducing dimensions", Sensors and Actuators B, vol 108, n¹-2, pp. 979-985, 2005.

[2] D. Lange, C. Hagleitner, A. Hierlemann , O. Brand and H. Baltes, "Complementary metal oxide semiconductor cantilever arrays on a single chip: mass-sensitive detection of volatile organic compounds", Analytical Chemistry, vol. 74, pp. 3084-3095, 2002.

[3] J.E. Sader, "Frequency response of cantilever beams immersed in viscous fluids with applications to the atomic force microscope", Journal of Applied Physics, 84, pp.64-76, 1998.

[4] U. Sampath, "Analytical Modeling of Microcantilever-Based Dynamic Microsensors," M.S. Thesis, Marquette University, Milwaukee, WI, 2005.

[5] K.Y. Yasumura, T.D. Stowe, E.M. Chow, T. Pfafman, T.W. Kenny, B.C. Stipe and D. Rugar, "Quality factors in micron- and submicronthick cantilevers", Journal of microelectromechanical systems, 9, pp. 117-125, 2000

[6] P. Lu, F. Shen, S.J. O'Shea, K.H. Lee and T.Y. Ng, "Analysis of surface effects on mechanical properties of microcantilevers", Materials Physics and Mechanics, vol. 4, pp. 51-55, 2001.

[7] Ferry, J.D.: Viscoelastic properties of polymers, John Wiley and Sons, 1961.

[8] Polymer data handbook, Oxford University Press, 1999.

[9] N.V. Lavrik, M.J. Sepaniak and P.G. Datskos, "Cantilever transducers as a platform for chemical and biological sensors", Review of Scientific Instruments, 75, pp. 2229-2253, 2004.

[10] L. Fadel, I. Dufour, F. Lochon and O. Français, Signal-to-noise ratio of resonant microcantilever type chemical sensors as a function of resonant frequency and quality factor, Sensors and Actuators B, 99, pp. $73-77,2004$ 